\title{
Nonlinear Dynamics for Gear Fault Level
}

\author{
Su Xunwen ${ }^{*}$, , Liu Jinhao ${ }^{1}$ and Wang Shaoping ${ }^{2}$ \\ ${ }^{1}$ School of Technology, Beijing Forestry University, Beijing, 100083, China \\ ${ }^{2}$ School of Automation Science and Electrical Engineering, Beijing University of Aeronautics and Astronautics, Beijing, \\ 100191, China
}

\begin{abstract}
Since there are backlash friction and time-varying stiffness, the characteristics of gear pair has strong nonlinearity. When a gear has faults such as pitting, spalling and tooth breakage, there are often some nonlinear characteristics as super-harmonics or sub-harmonics along with vibration signal. These characteristics will cause some difficulty for fault diagnostics, so it is necessary to study the mechanism of nonlinear vibration. Fourier expression was used to describe time-varying stiffness, a factor was used to depict gear fault level and nonlinear dynamics model for gear fault level was established. Simulation results show that, with certain friction and rotating speed, the nonlinear vibration has direct relationship with rotating speed and gear friction and has no direct relationship with fault types.
\end{abstract}

Keywords: Dynamics, fault diagnostics, fault level, gear, nonlinearity.

\section{INTRODUCTION}

Much work has been done since the first paper on gear vibration had been presented in 1920 [1]. The gear vibration has strong nonlinearity because of teeth friction, backlash and time-varying stiffness when the gear pair works normally [2-6]. As analytic solutions of the nonlinear vibration equation cannot be obtained, Japanese researcher got the linear approximate equation by doing some linear work on the equation and verified it by experiments [7]. Obviously, when a gear has faults such as pitting, spalling or a broken tooth and so on, the fault source will lead the timevarying stiffness to vary severely, and that is the most important reason leading to aggravate nonlinear vibration of gear pair. When a gear has faults, there are often some nonlinear phenomena such as super-harmonics and subharmonics. However, up till now, the research on mechanism of nonlinear vibration of gear fault is very few, the fault diagnostics of a gear is more concentrated on methods of signal processing, such as time domain analysis, frequency domain analysis, time-frequency joint domain analysis, etc. $[8,9]$. In [10], the authors investigated the phenomena of cycle vibration and chaos excited by the piecewise continuous stiffness and depicted fault gear stiffness using the piecewise continuous function. However that article does not consider the influences of friction and backlash which is rather significant. In addition, there is no shock phenomenon in the simulation result and it has not been verified by experiments.

Since the characteristics of gear pair and meshing stiffness have vast changes, so the nonlinear characteristics of the gear fault signal perform more significantly when a

*Address correspondence to this author at the School of Technology, Beijing Forestry University, No.35 Tsinghua East Road, Haidian District, Beijing, 100083, China; Tel: 86-10-62338153;

E-mail: suxw0703@gmail.com gear has fault. It is therefore, necessary to study the nonlinearity of a fault gear, especially the steady-state response of the faulty gear's nonlinear vibration to help the gear's fault diagnostics. The nonlinear dynamics integrated model of normal gears and fault gears is established considering friction, backlash and time-varying stiffness. Fourier series is used to depict varying stiffness, a gear fault factor is induced to depict gear fault level and simulation results are also presented. Analysis on characteristics of the gear box vibration shows that simulation results are consistent with experimental results.

\section{NONLINEAR DYNAMICS MODEL FOR A NORMAL GEAR PAIR CONSIDERING FRICTION AND BACKLASH}

The nonlinear dynamics model of a gear pair is shown in Fig. (1) considering friction and backlash.

The nonlinear dynamics, according to works of $[2,11]$ with friction and backlash of the gear pair are as follows.

$$
\begin{aligned}
& I_{p} \ddot{\theta}_{p}+r_{p}\left[c_{m} \dot{x}+k_{m}(t) f(x)\right]+l_{p} \lambda \mu\left[c_{m} \dot{x}+k_{m}(t) f(x)\right]=T_{p} \\
& I_{g} \ddot{\theta}_{g}-r_{g}\left[c_{m} \dot{x}+k_{m}(t) f(x)\right]-l_{g} \lambda \mu\left[c_{m} \dot{x}+k_{m}(t) f(x)\right]=-T_{g}
\end{aligned}
$$

Here,

$$
\begin{aligned}
& l_{p}=r_{p} \tan \beta+y, l_{g}=\sqrt{a^{2}-\left(r_{p}+r_{g}\right)^{2}}-l_{p}, \omega_{h}=z_{p} \omega_{p}, \\
& y=r_{p} \theta_{p}, k_{m}=k_{a v}+k_{a} \sin \left(\omega_{h} t\right), e(t)=e_{a} \sin \omega_{h} t, \\
& x=r_{p} \theta_{p}-r_{g} \theta_{g}-e(t), \lambda=\operatorname{sgn}\left[\left(\omega_{p}+\dot{\theta}_{p}\right) l_{p}-\left(\omega_{g}+\dot{\theta}_{g}\right) l_{g}\right], \\
& f(x)=\left\{\begin{array}{cc}
x-b, & x>b \\
0, & |x| \leq b \\
x+b, & x<-b
\end{array}\right.
\end{aligned}
$$




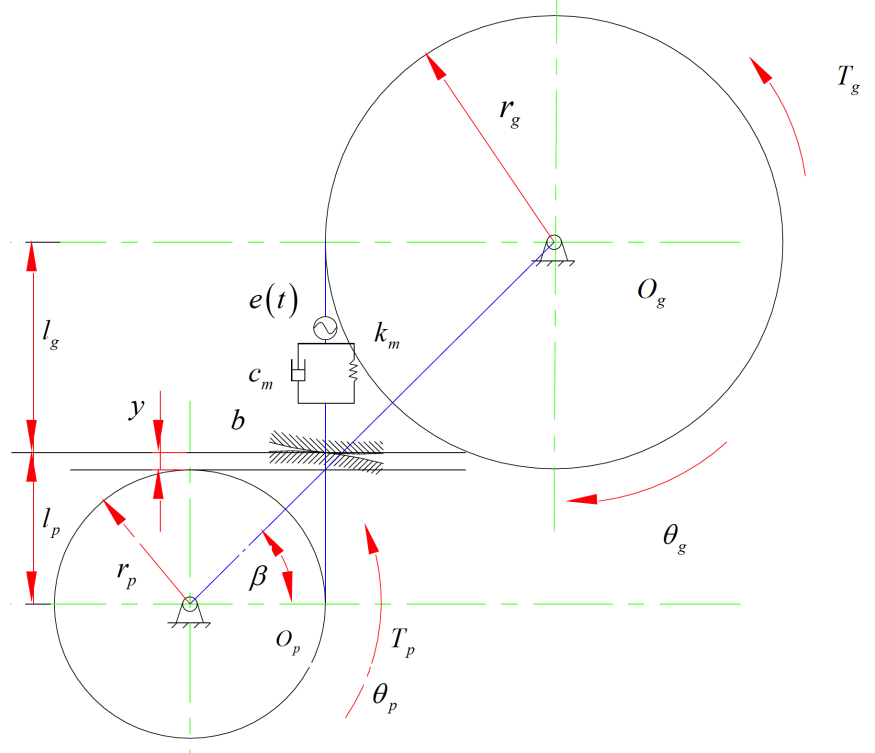

Fig. (1). Nonlinear dynamics model of a gear pair considering friction and backlash.

Eq. (1). $\times r^{r} / I_{p}-$ Eq. (2). $\times r_{g} / I_{g}-\ddot{e}$, and multiply with $1 / b \omega_{e}^{2}$, we have,

$\frac{\ddot{x}}{b \omega_{e}^{2}}+\left(\frac{r_{p}^{2}}{I_{p}}+\frac{r_{g}^{2}}{I_{g}}+\frac{l_{p} \lambda \mu r_{p}}{I_{p}}+\frac{l_{g} \lambda \mu r_{g}}{I_{g}}\right) \frac{c_{m} \dot{x}}{b \omega_{e}^{2}}+$

$\left(\frac{r_{p}^{2}}{I_{p}}+\frac{r_{g}^{2}}{I_{g}}+\frac{l_{p} \lambda \mu r_{p}}{I_{p}}+\frac{l_{g} \lambda \mu r_{g}}{I_{g}}\right) \frac{k_{m}(t) f(x)}{b \omega_{e}^{2}}$

$=\frac{T_{p} r_{p}}{I_{p} b \omega_{e}^{2}}+\frac{T_{g} r_{g}}{I_{g} b \omega_{e}^{2}}-\frac{\ddot{e}(t)}{b \omega_{e}^{2}}$

Eq. (1). $\times r_{p} /\left(I_{p} \omega_{e}^{2}\right)$ and simplifying it, we have,

$\frac{r_{p} \ddot{\theta}_{p}}{\omega_{e}^{2}}+\left(1+\frac{l_{p} \lambda \mu}{r_{p}}\right) \frac{r_{p}^{2} c_{m} \dot{x}}{I_{p} \omega_{e}^{2}}+\left(1+\frac{l_{p} \lambda \mu}{r_{p}}\right) \frac{r_{p}^{2} k_{m}(t) f(x)}{I_{p} \omega_{e}^{2}}=\frac{T_{m} r_{p}}{I_{p} \omega_{e}^{2}}$

Deal with Eq. (3) and Eq. (4) with non-dimension, let,

$$
\begin{aligned}
& \bar{x}=x / b, \bar{y}=y / b, m_{e}=m_{p} m_{g} /\left(m_{p}+m_{g}\right), m_{p}=I_{p} / r_{p}^{2}, \\
& m_{g}=I_{g} / r_{g}^{2}, \omega_{e}=\sqrt{k_{a v} / m_{e}}, \omega_{1 p}=\sqrt{k_{a v} / m_{p}}, \bar{\omega}=\omega_{h} / \omega_{e}, \\
& \tau=\omega_{e} t, \zeta_{1}=c_{m} /\left(2 m_{e} \omega_{e}\right), \\
& \zeta_{2}=c_{m} /\left(2 m_{p} \omega_{e}\right), k_{1}=k_{a} / k_{a v}, k_{2}=\omega_{p}^{2} / \omega_{e}^{2}, F_{a v}=T_{p} /\left(r_{p} m_{e} \omega_{e}^{2} b\right), \\
& F_{e}=e_{a} / b, f(\bar{x})=\left\{\begin{array}{cl}
\bar{x}-1, & \bar{x}>1 \\
0, & |\bar{x}| \leq 1 \\
\bar{x}+1, & \bar{x}<-1
\end{array}\right.
\end{aligned}
$$

Since, $\frac{d x}{d \tau}=\frac{d x}{d t} \times \frac{d t}{d \tau}$, it follows that $\dot{\bar{x}}(\tau)=\dot{\bar{x}}(t) \times \frac{1}{\omega_{e}}$,

$\ddot{\bar{x}}(\tau)=\ddot{\bar{x}}(t) \times \frac{1}{\omega_{e}^{2}}$,

Then, from Eq. (3) we obtain:

$$
\begin{aligned}
& \frac{\ddot{x}}{b \omega_{e}^{2}}=\frac{\ddot{x} / b}{\omega_{e}^{2}}=\frac{\ddot{\bar{x}}(t)}{\omega_{e}^{2}}=\ddot{\bar{x}}(\tau), \frac{\dot{x}}{b \omega_{e}^{2}}=\frac{\dot{x} / b}{\omega_{e}^{2}}=\frac{\dot{\bar{x}}(t)}{\omega_{e}^{2}}=\dot{\bar{x}}(\tau), \\
& \frac{k_{m}(t)}{\omega_{e}^{2} m_{e}}=\frac{\left(k_{a v}+k_{a} \sin \omega_{h} t\right)}{\omega_{e}^{2} m_{e}}=1+k_{1} \sin \left(\frac{\omega_{h}}{\omega_{e}} \omega_{e} t\right)=1+k_{1} \sin (\bar{\omega} \tau) \\
& \text { Since, } r_{p} \omega_{p}=r_{g} \omega_{g}, \lambda=\operatorname{sgn}\left[\left(\omega_{p}+\dot{\theta}_{p}\right) l_{p}-\left(\omega_{g}+\dot{\theta}_{g}\right) l_{g}\right],
\end{aligned}
$$

where,

$$
\begin{aligned}
& \left(\omega_{p}+\dot{\theta}_{p}\right) l_{p}-\left(\omega_{g}+\dot{\theta}_{g}\right) l_{g} \\
& =\omega_{e}\left[\frac{\left(r_{p} \omega_{p}+r_{p} \dot{\theta}_{p}\right)}{r_{p} \omega_{e}} l_{p}-\frac{\left(r_{g} \omega_{g}+r_{g} \dot{\theta}_{g}\right)}{r_{g} \omega_{e}} l_{g}\right]=\omega_{e} b_{h} \\
& {\left[\frac{\left(r_{p} \omega_{p} /\left(b_{h} \omega_{e}\right)+\dot{\bar{y}}(\tau)\right)}{r_{p}} l_{p}-\right.} \\
& \left.\frac{\left(r_{p} \omega_{p} /\left(b_{h} \omega_{e}\right)+\dot{\bar{y}}(\tau)-\dot{\bar{x}}(\tau)-F_{e} \bar{\omega} \cos (\bar{\omega} \tau)\right)}{r_{g}}\left(\sqrt{a^{2}-\left(r_{p}+r_{g}\right)^{2}}-l_{p}\right)\right]
\end{aligned}
$$

Since,

$$
\left(\frac{l_{p}}{m_{p} r_{p}}+\frac{l_{g}}{m_{g} r_{g}}\right) m_{e} \lambda=\left(\frac{\sqrt{a^{2}-\left(r_{p}+r_{g}\right)^{2}}}{m_{g} r_{g}}+\left(\frac{1}{m_{p} r_{p}}-\frac{1}{m_{g} r_{g}}\right) l_{p}\right) m_{e} \lambda
$$

Let $g_{1}(\tau)$ equals to Eq. (5). As known, $\frac{T_{p}}{r_{p}}=\frac{T_{g}}{r_{g}}$, since,

$\frac{T_{p}}{m_{p} r_{p} b \omega_{e}^{2}}+\frac{T_{g}}{m_{g} r_{g} b \omega_{e}^{2}}-\frac{\ddot{e}(\tau)}{b}=\frac{T_{p}}{r_{p} b \omega_{e}^{2}}\left(\frac{1}{m_{p}}+\frac{1}{m_{g}}\right)-\frac{\ddot{e}(\tau)}{b}=$ $\frac{T_{p}}{r_{p} b \omega_{e}^{2} m_{e}}-\frac{\ddot{e}(\tau)}{b}=F_{a v}-\frac{\ddot{e}(\tau)}{b}$

$e(t)=e_{a} \sin \omega_{h} t=e_{a} \sin \left(\frac{\omega_{h}}{\omega_{e}} \omega_{e} t\right)=e_{a} \sin \bar{\omega} \tau \quad, \quad$ Then, $e(\tau)=e_{a} \sin \bar{\omega} \tau, \ddot{e}(\tau) / b=-e_{a} \bar{\omega}^{2} \sin \bar{\omega} \tau / b=-F_{e} \bar{\omega}^{2} \sin \bar{\omega} \tau$,

Then, Eq. (3) is simplified at last as below,

$$
\begin{aligned}
& \ddot{\bar{x}}(\tau)+2 \zeta_{1}\left[1+g_{1}(\tau) \mu\right] \dot{\bar{x}}(\tau)+\left[1+g_{1}(\tau) \mu\right]\left[1+k_{1} \sin (\bar{\omega} \tau)\right] f(\bar{x}) \\
& =F_{a v}+F_{e} \bar{\omega}^{2} \sin \bar{\omega} \tau
\end{aligned}
$$

Let

$$
g_{2}(\tau)=\frac{l_{p} \lambda}{r_{p}}
$$




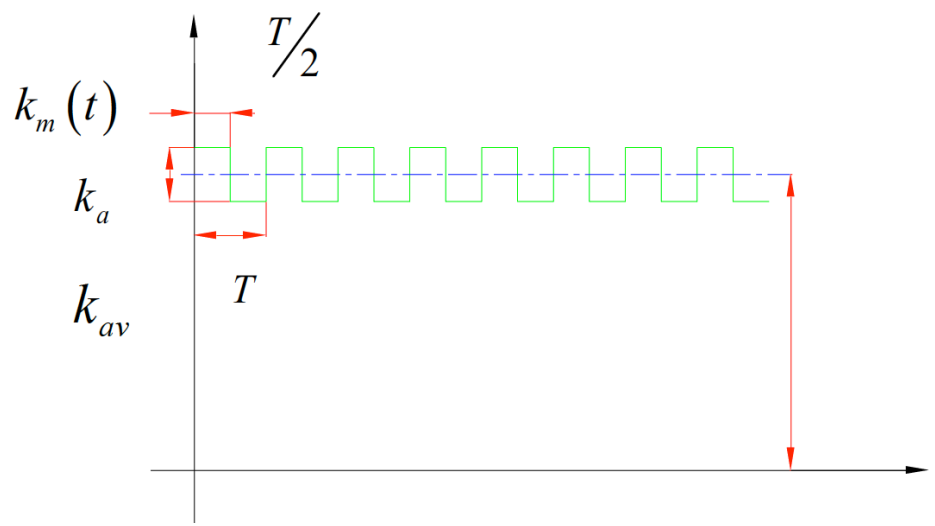

$T$ Meshing period; $k_{a}$ Peak-to-peak value of meshing stiffness; $k_{a v}$ Mean of meshing stiffness

Fig. (2). Meshing stiffness curve of a normal gear pair.

Substituting above equation to Eq. (4), and the necessary simplification, we obtain,

$\ddot{\bar{y}}(\tau)+2 \zeta_{2}\left[1+g_{2}(\tau) \mu\right] \dot{\bar{x}}(\tau)$

$+\left[1+g_{2}(\tau) \mu\right] k_{2}\left(1+k_{1} \sin \bar{\omega} \tau\right) f(\bar{x})=F_{a v} m_{e} / m_{1}$

Now the non-dimensional equations of the gear pair nonlinear dynamics are obtained as below,

$$
\left\{\begin{array}{l}
\ddot{\bar{x}}(\tau)+2 \zeta_{1}\left[1+g_{1}(\tau) \mu\right] \dot{\bar{x}}(\tau)+ \\
{\left[1+g_{1}(\tau) \mu\right]\left[1+k_{1} \sin (\bar{\omega} \tau)\right] f(\bar{x})=F_{a v}+F_{e} \bar{\omega}^{2} \sin \bar{\omega} \tau} \\
\quad \ddot{\bar{y}}(\tau)+2 \zeta_{2}\left[1+g_{2}(\tau) \mu\right] \dot{\bar{x}}(\tau)+ \\
\quad\left[1+g_{2}(\tau) \mu\right] k_{2}\left(1+k_{1} \sin \bar{\omega} \tau\right) f(\bar{x})=F_{a v} m_{e} / m_{1}
\end{array}\right.
$$

\section{NONLINEAR DYNAMICS MODEL OF A FAULT GEAR PAIR CONSIDERING FRICTION AND BACKLASH}

\subsection{Time-Varying Meshing Stiffness of a Fault Gear}

When a gear has faults such as pitting or broken tooth, the characteristics of meshing stiffness will change [12], in particular, when a tooth is broken, the variation of the meshing stiffness is very profound. Fig. (2) depicts timevarying stiffness of a normal gear, supposing each tooth has the same variance $[6,9]$.

When a tooth of a gear has fault, there must be reduction of meshing stiffness of the corresponding tooth meshing. Suppose a gear has teeth number equal to $Z$, and there is one broken tooth, the time-varying meshing stiffness coincides with Fig. (2). Suppose the broken tooth has the same peakto-peak value as that of normal teeth.

The Fourier series of the signal $F(t)$ in Fig. (2) is equal to,

$$
\begin{aligned}
& k_{m}(t)=k_{a v}+\frac{a_{0}}{2}+\sum_{n=1}^{\infty}\left(a_{n} \cos n \omega_{h} t+b_{n} \sin n \omega_{h} t\right) \\
& =k_{a v}+\frac{2 k_{a}}{\pi n} \sum_{n=1}^{\infty} \sin n \omega_{h} t, n=1,3,5, \ldots
\end{aligned}
$$

where,

$$
a_{0}=\frac{2}{T} \int_{0}^{T} F(t) d t=0 \quad, \quad a_{n}=\frac{2}{T} \int_{0}^{T} F(t) \cos (n \omega t) d t=0,
$$

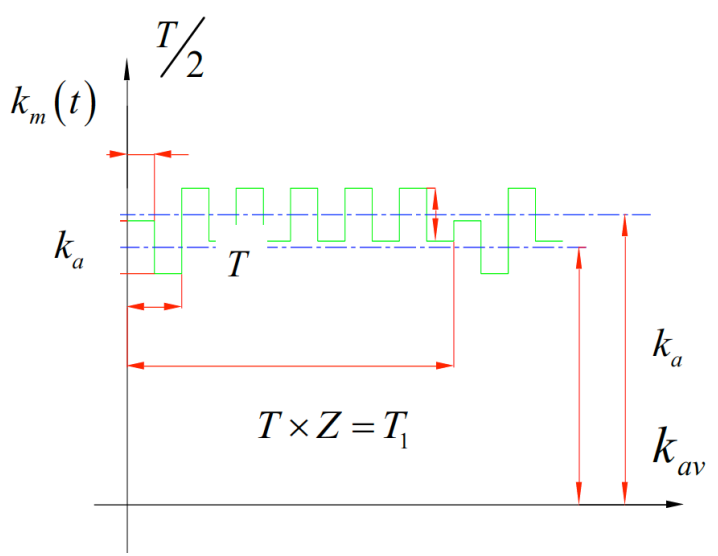

$T$ Meshing period; $T_{1}$ Rotational period; $Z$ Teeth of driving gear; $k_{a}$ Peak-to-peak value of meshing stiffness; $k_{a v}$ Mean of meshing stiffness; $k_{a v 1}$ Mean of meshing stiffness of the broken tooth

Fig. (3). Variety of meshing stiffness of a single broken tooth. 


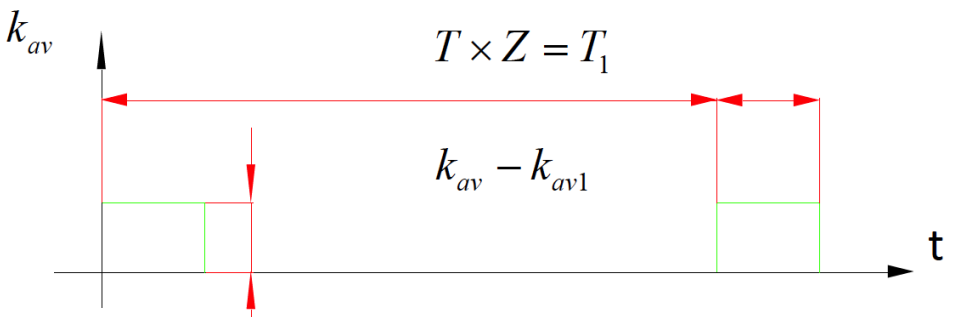

Fig. (4). Peak-to-peak value of the meshing stiffness of a broken tooth meshing.

$b_{n}=\frac{2}{T} \int_{0}^{T} F(t) \sin (n \omega t) d t=\frac{4 k_{a}}{\pi n}, n=1,3,5, \ldots, \omega_{h}=2 \pi / T$,

The below is to obtain the parameters of the Fourier series $k_{m}(t)$ of the signal $F(t)$ in Fig. (2).

Fig. (4) depicts the peak-to-peak value of the meshing stiffness of a broken tooth meshing. It can be seen that the Fig. (4) is the difference of Figs. $(\mathbf{2}, \mathbf{3})$.

Let. Since,

$F(t)=\frac{a_{0}}{2}+\sum_{n=1}^{\infty}\left(a_{n} \cos n \omega_{p} t+b_{n} \sin n \omega_{p} t\right)$,

$a_{0}=\frac{2}{T_{1}} \int_{0}^{T_{1}} F(t) d t=\frac{2 F_{0}}{Z}$,

$a_{n}=\frac{2}{T_{1}} \int_{0}^{T_{1}} F(t) \cos (n \omega t) d t=\frac{F_{0}}{n \pi} \sin (2 n \pi / Z)$,

$b_{n}=\frac{2}{T_{1}} \int_{0}^{T_{1}} F(t) \sin (n \omega t) d t=\frac{F_{0}}{\pi n}[1-\cos (2 n \pi / Z)]$
Then, the Fourier translation of Fig. (4) is obtained as below,

$k(t)=\frac{2 k_{b}}{Z}+$

$\sum_{n=1}^{\infty}\left\{\frac{k_{b}}{n \pi} \sin (2 n \pi / Z) \cos n \omega_{p} t+\frac{k_{b}}{\pi n}\left[1-\cos (2 n \pi / Z) \sin n \omega_{p} t\right]\right\}$

Then the expression of the meshing stiffness of a gear pair with a broken tooth fault, or the Fourier transmission of Fig. (3) is equal to,

$k_{m f}(t)=k_{m}(t)-k(t)=k_{a v}+\left\{\frac{2 k_{a}}{\pi n} \sum_{n=1}^{\infty} \sin n \omega_{h} t, n=1,3,5, \ldots\right\}-\frac{2 k_{b}}{Z}$

$+\sum_{n=1}^{\infty}\left\{\frac{k_{b}}{n \pi} \sin (2 n \pi / Z) \cos n \omega_{p} t+\frac{k_{b}}{\pi n}\left[1-\cos (2 n \pi / Z) \sin n \omega_{p} t\right]\right\}$

The curves in Figs. (5-7) are the simulation results of Eq. (10) to Eq. (12), respectively using Matlab. The meshing period is $T=20 / 1024 \mathrm{Sec}$; teeth of the gear is $Z=6$; mean of meshing stiffness is $k_{a v}=20$; peak-to-peak value of meshing stiffness is $k_{a}=0.5$; with the maximum Fourier order of

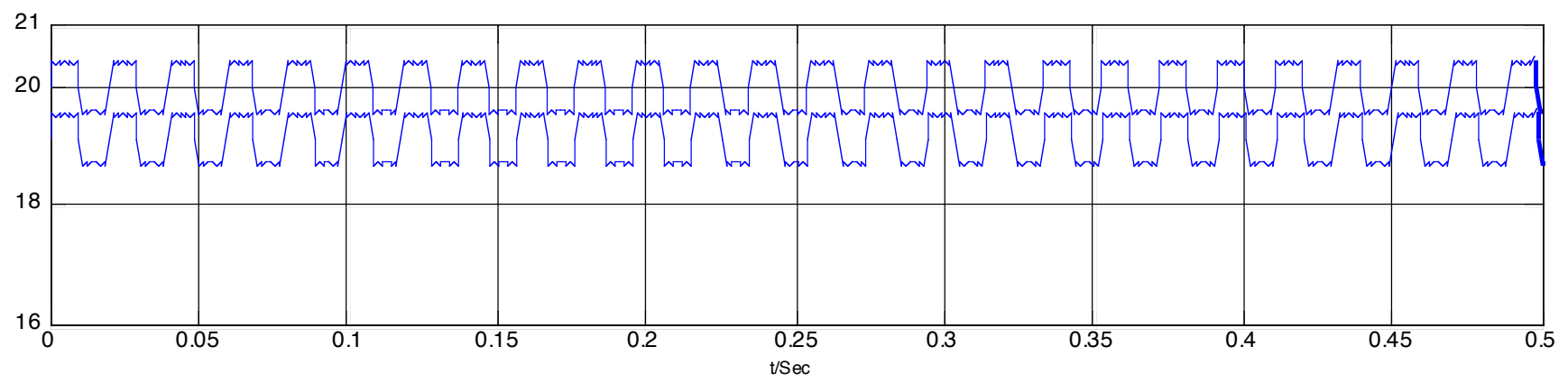

Fig. (5). Time-varying meshing stiffness of a normal gear.

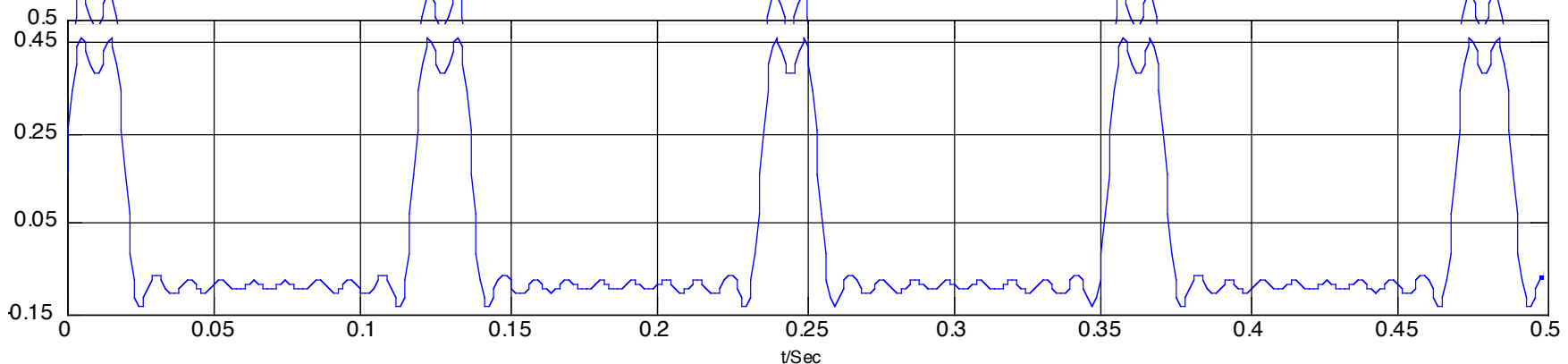

Fig. (6). Time-varying meshing stiffness of a broken tooth meshing. 


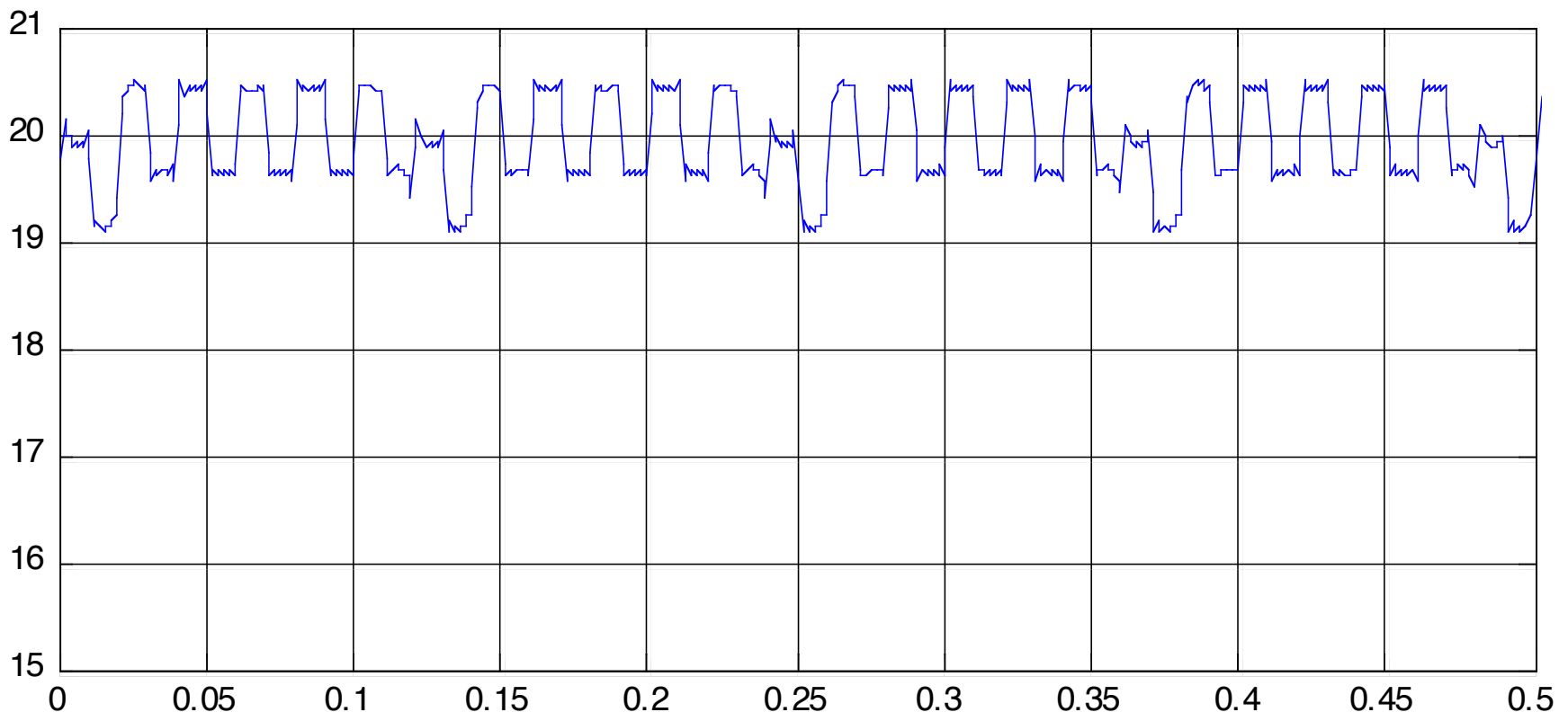

Fig. (7). Time-varying meshing stiffness of a gear with fault of a broken tooth.

$n_{\max }=10$.

From Fig. (7), it can be seen that the curve of Fig. (7) can express the time-varying stiffness of a fault gear in Fig. (3). approximately.

\subsection{Nonlinear Dynamics Integrated Model}

Since there will be variety of gear faults such as pitting and spalling and so on, and the variation of the meshing stiffness is less than that of broken tooth fault, transmit Eq. (12) as below,

$$
\begin{aligned}
& k_{m f}(t)=k_{m}(t)-\alpha k_{1}(t) \\
& =k_{a v}\left\{1+\left\{\frac{2 k_{a}}{k_{a v} \pi n} \sum_{n=1}^{\infty} \sin n \omega_{h} t, n=1,3,5, \ldots\right\}\right\}-\alpha k_{a v} \\
& \left\{\begin{array}{l}
\frac{2 k_{b}}{Z k_{a v}}+\sum_{n=1}^{\infty}\left\{\begin{array}{l}
\frac{k_{b}}{n \pi k_{a v}} \sin (2 n \pi / Z) \cos n \omega_{p} t \\
+\frac{k_{b}}{\pi n k_{a v}}\left[1-\cos (2 n \pi / Z) \sin n \omega_{p} t\right]
\end{array}\right\}
\end{array}\right\} \\
& =k_{a v}\left\{1+\left\{\frac{2 k_{1}}{\pi n} \sum_{n=1}^{\infty} \sin n \omega_{h} t, n=1,3,5, \ldots\right\}\right\} \\
& -\alpha k_{a v}\left\{\frac{2 k_{3}}{Z}+\sum_{n=1}^{\infty}\left\{\begin{array}{l}
\frac{k_{3}}{n \pi} \sin (2 n \pi / Z) \cos n \omega_{p} t \\
+\frac{k_{3}}{\pi n}\left[1-\cos (2 n \pi / Z) \sin n \omega_{p} t\right]
\end{array}\right\}\right\}
\end{aligned}
$$

where,

$k_{1}=\frac{k_{a}}{k_{a v}}, k_{3}=\frac{k_{b}}{k_{a v}}$

$\alpha \in[0,1]$ is gear fault factor, when a gear is in normal status, $\alpha=0$; when a gear has serious fault of broken tooth, $\alpha=1$; otherwise when a gear has light faults such as crack, pitting, and spalling $0<\alpha<1$.

Dividing Eq. (13) by $\omega_{e}^{2} m_{e}$, then by simplifying it into its non-dimensional form, we get,

$$
\begin{aligned}
& \frac{k_{m f}(t)}{\omega_{e}^{2} m_{e}}=1+\left\{\frac{2 k_{1}}{\pi n} \sum_{n=1}^{\infty} \sin \left(n \frac{\omega_{h}}{\omega_{e}} \omega_{e} t\right), n=1,3,5, \ldots\right\} \\
& -\alpha\left\{\frac{2 k_{3}}{Z}+\sum_{n=1}^{\infty}\left\{\begin{array}{l}
\frac{k_{3}}{n \pi} \sin (2 n \pi / Z) \cos n \omega_{p} t \\
+\frac{k_{3}}{\pi n}\left[1-\cos (2 n \pi / Z) \sin n \omega_{p} t\right]
\end{array}\right\}\right\} \\
& =1+\left\{\frac{2 k_{1}}{\pi n} \sum_{n=1}^{\infty} \sin (n \bar{\omega} \tau), n=1,3,5, \ldots\right\}- \\
& \alpha\left\{\frac{2 k_{3}}{Z}+\sum_{n=1}^{\infty}\left\{\begin{array}{l}
\frac{k_{3}}{n \pi} \sin (2 n \pi / Z) \cos \left(n \omega_{p} \tau / \omega_{e}\right) \\
+\frac{k_{3}}{\pi n}\left[1-\cos (2 n \pi / Z) \sin \left(n \omega_{p} \tau / \omega_{e}\right)\right]
\end{array}\right\}\right\} \\
& =1+\left\{\frac{2 k_{1}}{\pi n} \sum_{n=1}^{\infty} \sin (n \bar{\omega} \tau), n=1,3,5, \ldots\right\}- \\
& \alpha\left\{\frac{2 k_{3}}{Z}+\sum_{n=1}^{\infty}\left\{\begin{array}{l}
\frac{k_{3}}{n \pi} \sin (2 n \pi / Z) \cos \left(n \tau \sqrt{k_{2}}\right) \\
+\frac{k_{3}}{\pi n}\left[1-\cos (2 n \pi / Z) \sin \left(n \tau \sqrt{k_{2}}\right)\right]
\end{array}\right\}\right\} \\
& \text { Let, } k_{11}=\frac{2 k_{1}}{\pi n} \sum_{n=1}^{\infty} \sin (n \bar{\omega} \tau), n=1,3,5, \ldots \\
& k_{22}=\frac{2 k_{3}}{Z}+\sum_{n=1}^{\infty}\left\{\frac{k_{3}}{n \pi} \sin (2 n \pi / Z) \cos n \tau+\frac{k_{3}}{\pi n}[1-\cos (2 n \pi / Z) \sin n \tau]\right\} \text {, }
\end{aligned}
$$


Then Eq. (16) can be simplified into, $\frac{k_{m f}(t)}{\omega_{e}^{2} m_{e}}=1+k_{11}-\alpha k_{12}$.

Therefore, from Eq. (1) and Eq. (2) the nonlinear dynamics integrated model of a normal gear and a fault gear, considering backlash and friction, is satisfied,

$$
\left\{\begin{array}{c}
I_{p} \ddot{\theta}_{p}+r_{b 1}\left[c_{m} \dot{x}+k_{m f}(t) f(x)\right]+l_{p} \lambda \mu\left[c_{m} \dot{x}+k_{m f}(t) f(x)\right]=T_{p} \\
I_{g} \ddot{\theta}_{g}-r_{g}\left[c_{m} \dot{x}+k_{m f}(t) f(x)\right]-l_{p} \lambda \mu\left[c_{m} \dot{x}+k_{m f}(t) f(x)\right]=-T_{g}
\end{array}\right.
$$

The Non-dimensional form is equal to,

$$
\left\{\begin{array}{l}
\ddot{\bar{x}}(\tau)+2 \zeta_{1}\left[1+g_{1}(\tau) \mu\right] \dot{\bar{x}}(\tau)+ \\
{\left[1+g_{1}(\tau) \mu\right]\left[1+k_{11}-\alpha k_{12}\right] f(\bar{x})=F_{a v}+F_{e} \bar{\omega}^{2} \sin \bar{\omega} \tau} \\
\quad \ddot{\bar{y}}(\tau)+2 \zeta_{2}\left[1+g_{2}(\tau) \mu\right] \dot{\bar{x}}(\tau)+ \\
\quad\left[1+g_{2}(\tau) \mu\right] k_{2}\left(1+k_{11}-\alpha k_{12}\right) f(\bar{x})=F_{a v} m_{e} / m_{1}
\end{array}\right.
$$

\section{SOLUTION}

Eq. (18) is a second order differential equation which can be solved by $4-5$ order Runge-Kutta method. In order to supply theoretical reference of gear faults diagnosis, the steady-state response of a normal gear and a fault gear vibration is focused as well on the frequency domain characteristics. For simplicity, the teeth of the driving gear are $z_{p}=6$, and the other parameters are as shown in Table 1. Parts of them are according to reference [2].

Given $\mu, \bar{\omega}$ and $\alpha$, the other parameters can be solved using parameters in Table 1. Non-dimensional frequency, $f_{n d}=\bar{\omega} / 2 \pi$.

Table 1. Parameter values.

\begin{tabular}{|c|c|c|c|}
\hline $\begin{array}{c}\text { Parameter } \\
\text { Symbols }\end{array}$ & Value & $\begin{array}{c}\text { Parameter } \\
\text { Symbols }\end{array}$ & Values \\
\hline \hline$z_{1}$ & 6 & $\zeta_{1}$ & 0.02 \\
\hline$a$ & $120 \mathrm{~mm}$ & $\zeta_{2}$ & 0.01 \\
\hline$b$ & $0.1 \mathrm{~mm}$ & $k_{1}$ & 0.2 \\
\hline$r_{p}$ & $56.382 \mathrm{~mm}$ & $k_{3}$ & 0.1 \\
\hline$r_{g}$ & $56.382 \mathrm{~mm}$ & $F_{a v}$ & 0.2 \\
\hline$r_{a g}$ & $63 \mathrm{~mm}$ & $F_{e}$ & 0.5 \\
\hline$I_{p}$ & $1.0 \mathrm{~kg} \cdot \mathrm{m}^{2}$ & $k_{a v}$ & $2.164 \times 10^{9}$ \\
\hline$I_{g}$ & $1.0 \mathrm{~kg} \cdot \mathrm{m}^{2}$ & & \\
\hline
\end{tabular}

Let $\mu=0.05, \bar{\omega}=0.3$, then, non-dimensional frequency is $f_{\text {nde }}=0.048$; non-dimensional period is $T_{\text {nde }}=20.9$; non-dimensional rotational period is $T_{n d}=125$; non-dimensional rotational frequency is $f_{n d}=0.008$. Steady-state response vibrations are shown in Figs. (8-10). with the frequency resolution of $\Delta f=0.0019$.

$\alpha=0$, this means the gear is in normal status. And Fig. (8) depicts the steady-state vibration response of a normal gear.

$\alpha=0.2$, this means the gear has some slight faults such as initial crack, pitting or spalling. Fig. (2). depicts the steady-state vibration response of a gear with fault of initial crack, pitting or spalling.

$\alpha=1$, this means the gear has serious faults as broken tooth. Fig. (10) depicts the steady-state vibration response of a gear with fault of broken tooth.

From the time domain signal of Fig. (10), it can be seen that the shock phenomenon is even more clear along with the increase of the fault factor $\alpha$, with shock period about 125 . Moreover, in the frequency domain signal of Fig. (10), the sidebands of $1 \mathrm{x}, 2 \mathrm{x}$ and high frequency multiplication of meshing frequency with an interval of about 0.008 are more and more clear.

$\mu=0, \bar{\omega}=0.7$, with non-dimensional meshing frequency $f_{\text {nde }}=0.1115$, non-dimensional meshing period $T_{\text {nde }}=9.0$

non-dimensional rotational period $T_{n d}=54$ and nondimensional rotational frequency $f_{n d}=0.019$. The steady-state vibration signals are shown in Figs. (11, 12) with frequency resolution $\Delta f=0.0019$.

$\alpha=0$, means the gear is in normal status. Fig. (11) depicts the steady-state vibration response of a normal gear.

$\alpha=1$, means the gear has serious faults as broken tooth. Fig. (12) depicts the steady-state vibration response of a gear with fault of broken tooth.

From Fig. (11), it can be seen that the normal gear vibration signal also has sidebands. However, when $\alpha=0$, there is no modulation frequency in the dynamics Eq. (18), so the sidebands should be superharmonics. In Fig. (11), the signal is about a gear with serious fault such as broken tooth, it is obvious that there are sidebands at the harmonics of meshing frequency with interval of rotational frequencies. Then, we conclude that when a gear is in nonlinear vibration, the super-harmonics are related with meshing frequency $\bar{\omega}$ and have nothing to do with gear faults. Fig. (11) reveals that the amplitudes of super-harmonics are much lower than that of sidebands of meshing frequency with gear faults.

\section{CONCLUSION}

The nonlinear dynamics integrated model of normal gears and fault gears has been established considering 

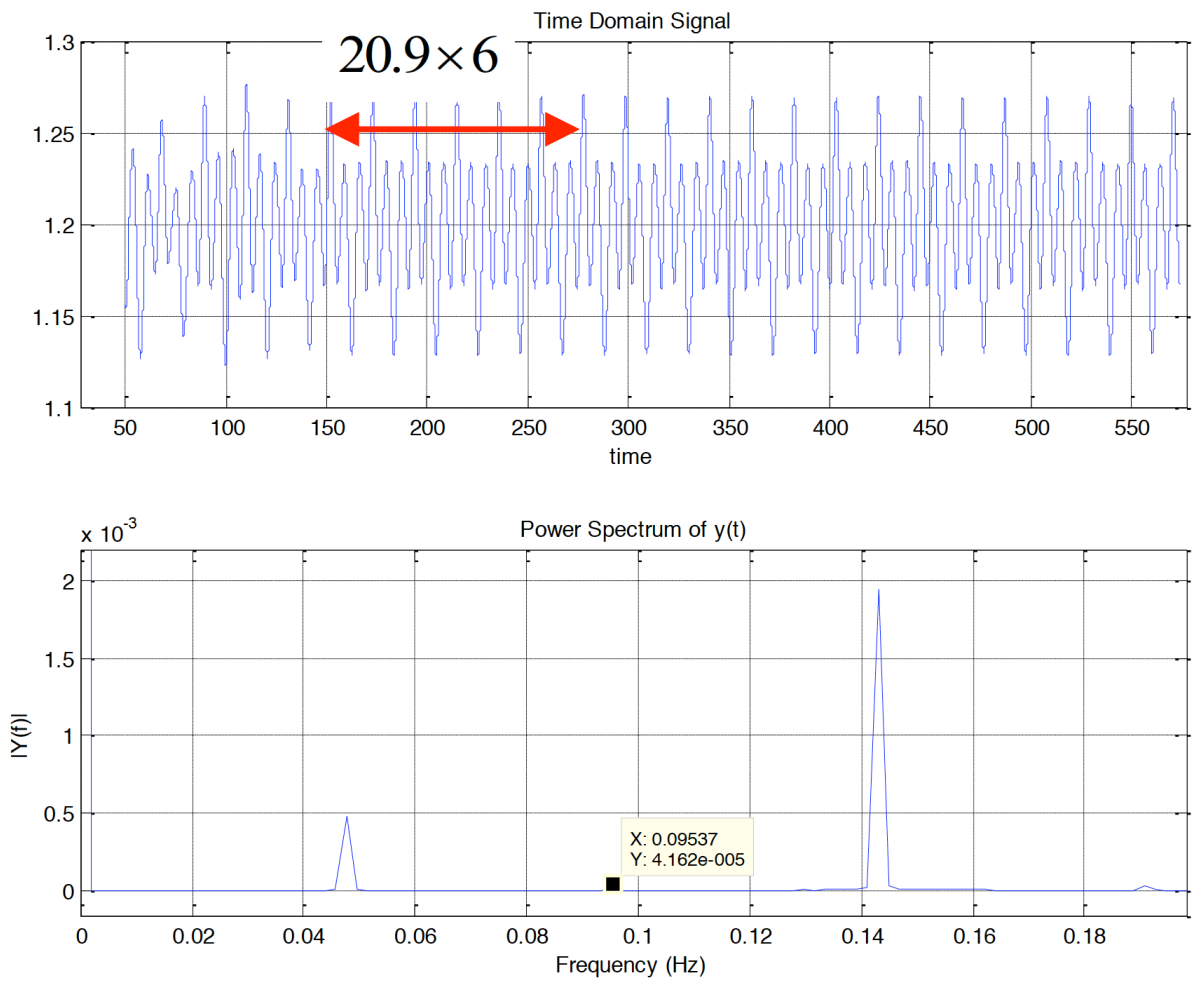

Fig. (8). Steady-state response of a normal gear vibration

\section{Time Domain Signal}
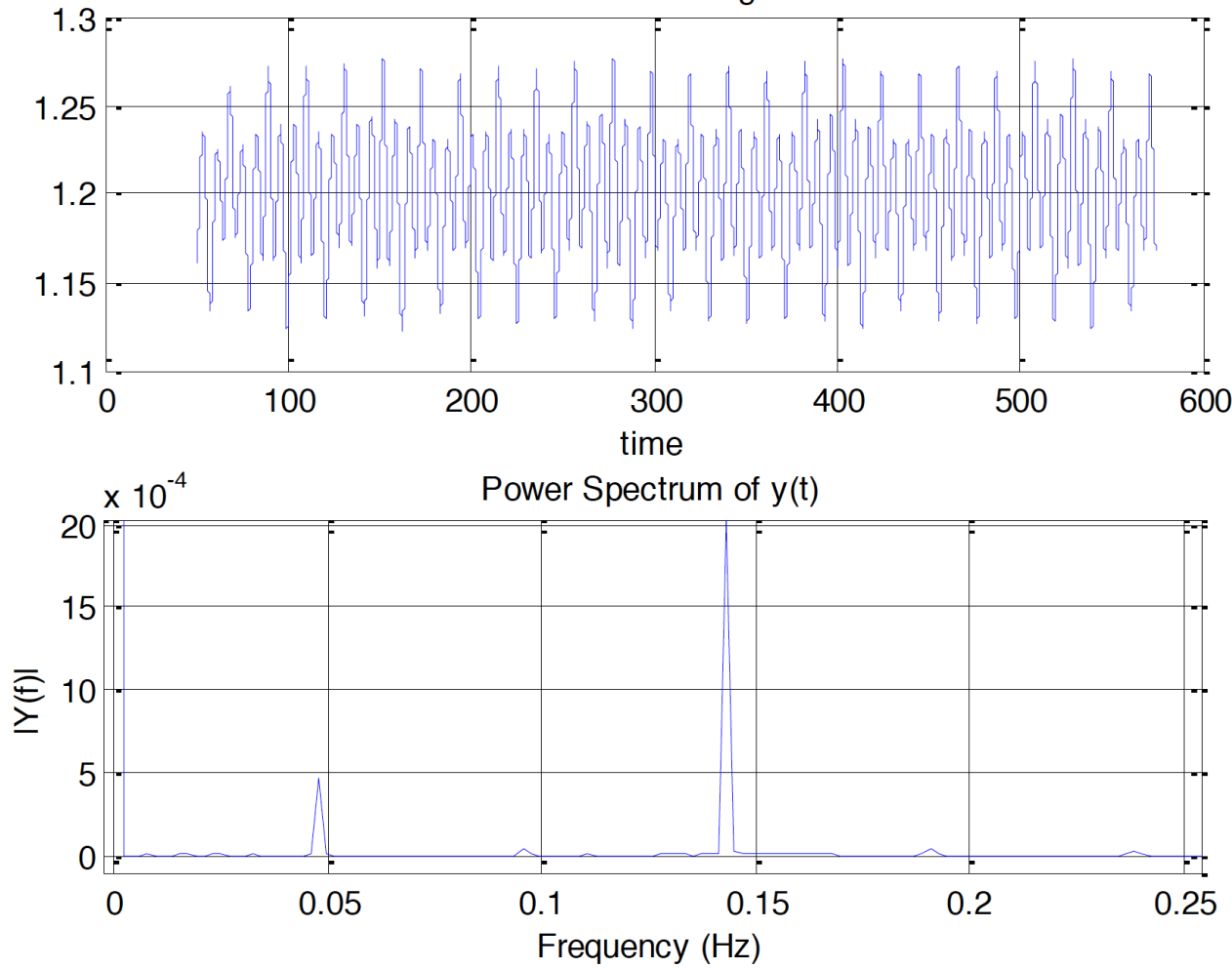

Fig. (9). Vibration response of a gear with fault of earlier crack, pitting or spalling.

friction, backlash and time-varying meshing stiffness. Fourier series is used to depict varying stiffness that is more suitable to solve the dynamics equation. The gear fault factor provides convenience for the integrated model to depict normal gear vibration and fault gear vibration. Simulations show that, 

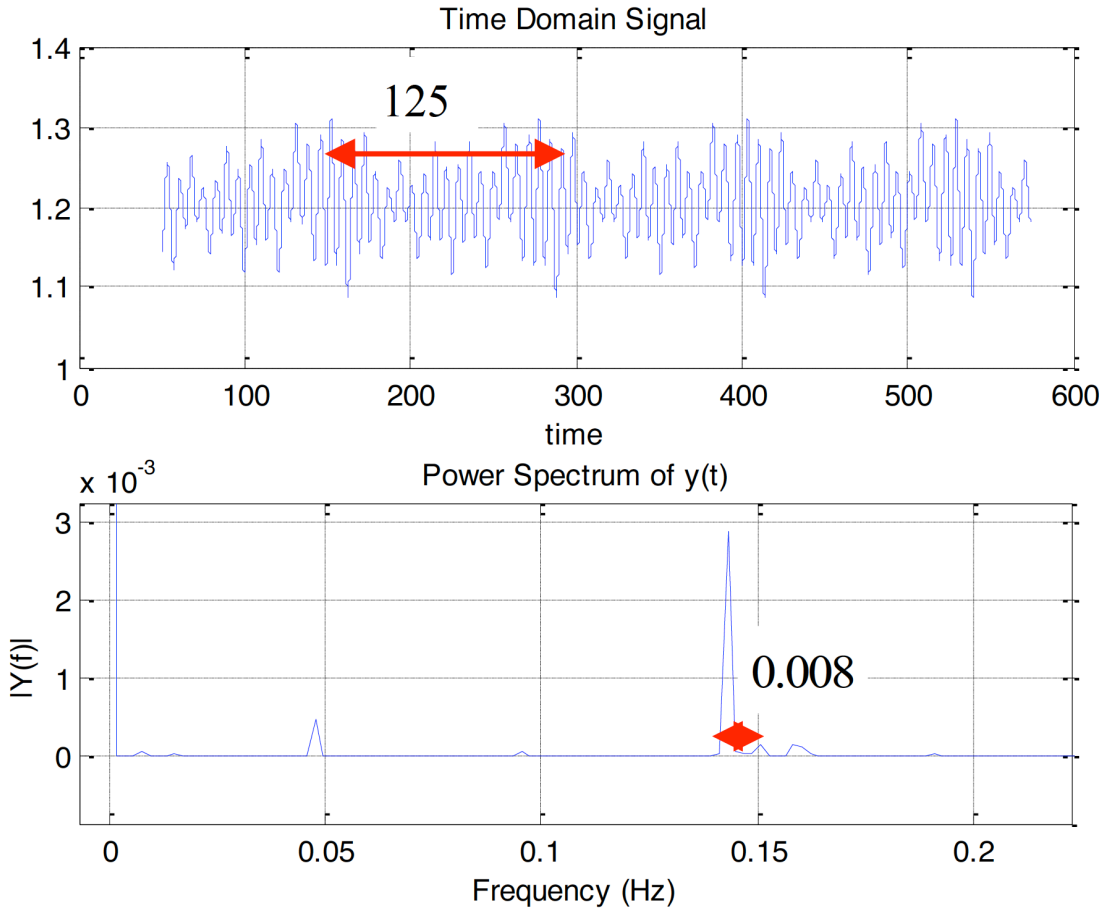

Fig. (10). Steady-state vibration response of a gear with fault of broken tooth.
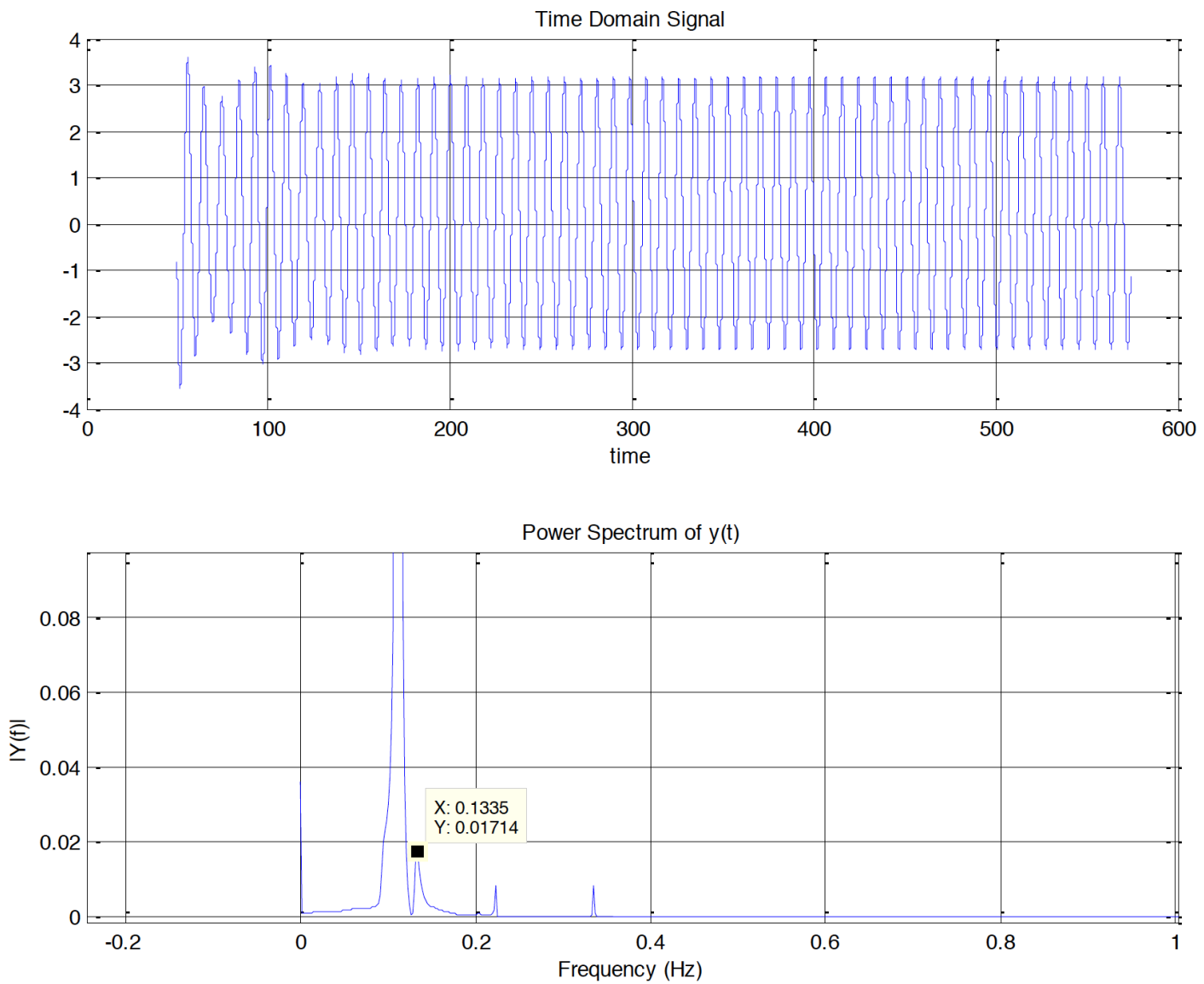

Fig. (11). Steady-state response of a normal gear vibration.

a. With the increase of the fault factor, which means the fault level is growing from light level to serious level, the shock phenomenon is more and more clear. Meshing frequency and its multiple frequency of the 


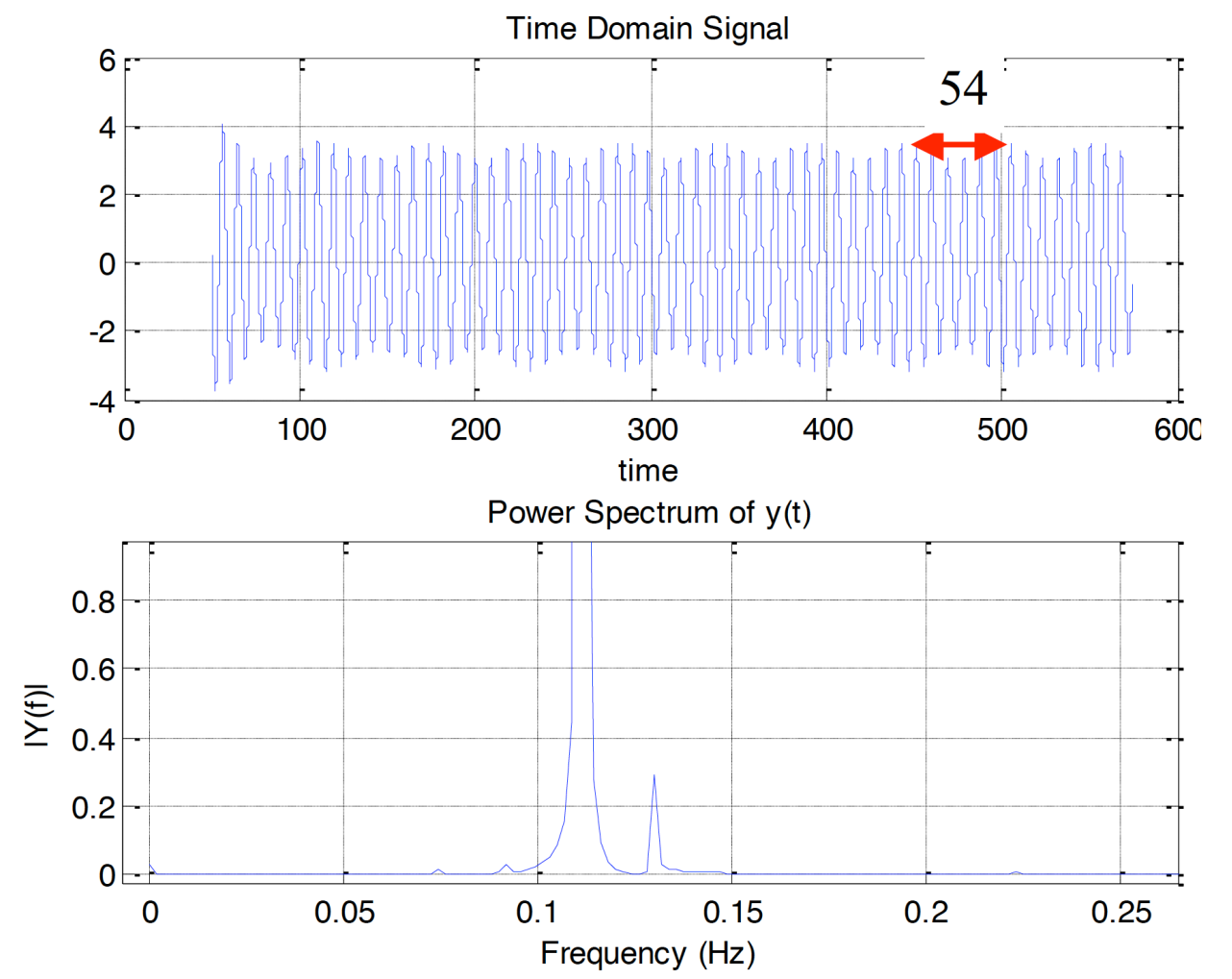

Fig. (12). Vibration response of a gear vibration with fault of a tooth broken.

vibration signal are modulated by the rotational frequency clearly that means the sidebands with interval of rotational frequency are more obvious.

b. Fault level of a gear is not the root cause leading to sub-harmonics or super-harmonics of gear vibration whose reason is related with rotational speed and friction coefficient of gear pair.

\section{NOMENCLATURE}

$I_{p}, I_{g}$

$\theta_{p}, \theta_{g}$

$r_{p}, r_{g}$

$l_{p}, l_{g}$

$T_{p}, T_{g}$

$m_{p}, m_{g}$

$\omega_{p}, \omega_{g}$

$O_{p}, O_{g}$

$z_{p}, z_{g}$

$x$
$=$ Moments of inertia of contacting tooth pairs

$=$ Rotational angles of contacting tooth pairs

$=$ Base radiuses of contacting tooth pairs

$y$

$\lambda$

$\mu$

$f(x)$

$c_{m}$

$k_{m}(t)$

$\beta$

$a$

$e(t)$

$=$ Friction arms of contacting tooth pairs

$=$ Torques of contacting tooth pairs

$=$ Masses of contacting tooth pairs

$=$ Angular velocities of contacting tooth pairs

$=$ Origin points of contacting tooth pairs

$=$ Teeth of contacting tooth pairs

$=$ Relative displacement between meshing points
$=$ Linear displacement of meshing point of driving gear

$=$ Direction coefficient of friction

$=$ Coefficient of kinetic friction

$=$ Backlash function

$=$ Meshing damping coefficient;

$=$ Meshing stiffness;

$=$ Pressure angle;

$=$ Center distance of contacting tooth pairs

$=$ Static error

$=$ Meshing time

$=$ Half of backlash

$=$ Mean of varying meshing stiffness

$=$ Peak-to-peak of varying meshing stiffness

$e_{a}(t) \quad=$ Amplitude of static error

$\omega_{h} \quad=$ Meshing frequency

$m_{e} \quad=$ Equivalent inertial mass of gear pair

$\omega_{e} \quad=$ Equivalent natural frequency of gear pair 


$$
\begin{aligned}
& \omega_{1 p} \quad=\text { Natural frequency of driving gear } \\
& \tau=\text { Non-dimensional time } \\
& \xi_{1}=\text { Damping ratio } \\
& \xi_{2} \quad=\text { Damping ratio } \\
& r_{a g} \quad=\text { Pitch Radius of driven gear } \\
& F_{a v} \quad=\text { Mean of non-dimensional exciting } \\
& F_{e} \quad=\text { Non-dimensional amplitude of } \\
& \text { transmission error } \\
& g_{1}(t), g_{2}(t) \quad=\text { Functions related friction direction to } \\
& \text { simplify dynamic equation } \\
& k_{1}, k_{2}, k_{3}, k_{11}, k_{22}=\text { Stiffness functions } \\
& \varepsilon \quad=\text { Contact ratio } \\
& \bar{x}, \bar{y}, \bar{\omega} \cdots \quad=\text { Corresponding non-dimensional } \\
& \text { symbols of } x, y, \omega \cdots \\
& k_{a v 1} \quad=\text { Mean of meshing stiffness } \\
& F(t) \quad=\text { Piecewise continuous function of time- } \\
& \text { varying meshing stiffness } \\
& T \quad=\text { Meshing period of gear pair } \\
& T_{1} \quad=\text { Rotation period of driving gear } \\
& Z \quad=\text { Teeth of driving gear } \\
& n_{\max } \quad=\text { The maximum number of Fourier series } \\
& f_{\text {nde }} \quad=\text { Non-dimensional meshing frequency } \\
& T_{n d e} \quad=\text { Non-dimensional meshing period } \\
& T_{n d} \quad=\text { Non-dimensional rotation period of } \\
& \text { driving gear } \\
& f_{n d} \quad=\text { Non-dimensional rotation frequency } \\
& \Delta f \quad=\text { Frequency resolution }
\end{aligned}
$$

\section{CONFLICT OF INTEREST}

We declare that this article content has no conflict of interest with other people or organizations that can inappropriately influence our work.

\section{ACKNOWLEDGEMENTS}

The authors would like to acknowledge the support from the Fundamental Research Funds for the Central Universities (YX2014-04).

\section{REFERENCES}

[1] A. Parey, and N. Tandon "Spur gear dynamic models including defects: A review", The Shock and Vibration Digest, vol. 35, pp.14-18, 2003.

[2] W. Sanmin, S. Yunwen, and D. Haijun, "Chaos and bifurcation analysis of a spur gear pair with combined friction and clearance", Chinese Journal of Mechanical Engineering, pp. 8-11, 2002.

[3] W. Jianjun, L. Qihan, and L. Runfang, "Research advances for nonlinear vibration of gear transmission systems", Advances in Mechanics, vol. 1, pp. 37-51, 2005.

[4] Y. Xianyong, Z. Xiaojun, and L. Yong, "Bifurcation and chaos of nonlinear system of helical bevel gear with clearance", Journal of Vibration and Shock, vol. 11, pp.115-9, 2008.

[5] J. Wang, and T. C. Lim. "Effect of tooth mesh stiffness asymmetric nonlinearity for drive and coast sides on hypoid gear dynamics", Journal of Sound and Vibration, vol. 319, pp. 885-903, 2009.

[6] F. Elasha, C. Ruiz-Cárcel, D. Mba, G. Kiat, I. Nze, and G. Yebra, "Pitting detection in worm gearboxes with vibration analysis", Engineering Failure Analysis, vol. 42, pp. 366-76, 2014.

[7] Y. Cai, and T. Hayashi, "The Linear approximated equation of vibration of a pair of spur gears (theory and experiment)", Journal of Mechanical Design, vol. 116, pp. 558-64, 1994.

[8] K. C. Gryllias, L. Gelman, B. Shaw, and M. Vaidhianathasamy, "Local damage diagnosis in gearboxes using novel wavelet technology", Insight, vol. 52, pp. 437-41, 2010.

[9] W. Bartelmus, F. Chaari, R. Zimroz, and M. Haddar, "Modelling of gearbox dynamics under time-varying nonstationary load for distributed fault detection and diagnosis", Europen Journal of Mechanics A-solids, vol. 29, pp. 637-46, 2010.

[10] G. Litak, and M. I. Friswell, "Dynamics of a gear system with faults in meshing stiffness", Nonlinear Dynamics, vol. 41, pp. 41521,2005 .

[11] L. Runfang, and W. Jianjun, Dynamics of gear system, Beijing: Science Press, 1997

[12] Y. Pandya, and A. Parey, "Crack behavior in a high contact ratio spur gear tooth and its effect on mesh stiffness", Engineering Failure Analysis, vol. 34, pp. 69-78, 2014. 